\title{
How effective is the use of glycemic variability in the follow-up of type 1 diabetes under intensive care?
}

\author{
Rodrigues R ${ }^{1}$, Medeiros $\mathrm{LA}^{2}$, Cunha LM², Garrote-Filho MS ${ }^{2}$, Neto MB ${ }^{3}$, Freitas MAR ${ }^{1}$, Jorge PT ${ }^{1}$, Resende ES ${ }^{1}$ and Penha-Silva N \\ ${ }^{1}$ Faculty of Medicine, Federal University of Uberlândia, MG, Brazil \\ ${ }^{2}$ Institute of Biotechnology, Federal University of Uberlândia, Uberlândia, MG, Brazil \\ ${ }^{3}$ Department of Basic and Environmental Sciences, University of São Paulo, Lorena, SP, Brazil
}

\begin{abstract}
Background: Glycemic variability (GV) has been identified as an important tool in the monitoring of type 1 diabetes mellitus (T1D), due to its ability to identify patients at greater risk of severe hypoglycemia and to constitute an independent risk factor for chronic complications of this disease. This study aimed to evaluate the GV pattern between individuals with T1D under different types of intensive treatment and the correlations of this GV with different clinical and biochemical variables.

Methods: Volunteers with T1D ( $\mathrm{n}=90$ ) under different types of intensive treatment (NPH insulin, $\mathrm{n}=54$; insulin glargine, $\mathrm{n}=19$; and continuous subcutaneous insulin infusion (CSII) system, $n=17$ ) were analyzed in relation to glycemic control and GV. The glycated hemoglobin (HbA1c) and estimated average glucose (eAG) were used to estimate glycemic control. The daily average glucose (DAG) and its standard deviation (SDDAG) and coefficient of variability (CVDAG), as well the change in the levels of glycated hemoglobin $(\triangle \mathrm{HbA} 1 \mathrm{c})$ were used to estimate $\mathrm{GV}$.

Results: Patients under CSII presented lower values of HbA1c, eAG, DAG, SDDAG and CVDAG than the other groups. DAG (median=189 mg/dL and IQR=157232) and SDDAG (median $=89.8 \mathrm{mg} / \mathrm{dL}$ and $\mathrm{IQR}=72-111$ ) correlated positively with $\mathrm{HbA1}$, with correlations coefficients of 0.67 and 0.64 , respectively.

Conclusions: The glycemic variability in patients with type 1 diabetes, even under intensive treatment, has a great amplitude, and has direct correlations with the classic parameters of disease control. DAG and SDDAG can be used in the short term as predictors of the glycated hemoglobin levels in order to allow directions and earlier adaptations in clinical management.
\end{abstract}

\begin{abstract}
Abbreviations: CGM: Continuous glucose monitoring; CSII:Continuous subcutaneous insulin infusion; CV: Coefficient of variability of daily average glucose; DAG: Daily average glucose; DDI: Daily dose of insulin; T1D: Type 1 diabetes mellitus; eAG: Estimated average glucose; FPG: Fasting plasma glucose; GV: Glycemic variability; HbAlc: Glycated hemoglobin; HBGI: High blood glucose index; LBGI: Low blood glucose index; MA: Microalbuminuria; SDDAG: Standard deviation of the daily average glucose; SMBG: Self-monitoring of blood glucose; $\triangle \mathrm{HbA1c}$ : HbAlc change.
\end{abstract}

\section{Introduction}

Besides the ability to predict the chronic complications of diabetes [1] glycemic variability (GV) may also allow identification of patients at higher risk of developing severe hypoglycemia [2,3]. This is why the approach of GV in the treatment of type 1 diabetes has been quite prominent in recent years. The development of new technologies to monitor blood glucose levels, such as the continuous interstitial glucose monitoring systems, have made possible and practical the evaluation of $\mathrm{GV}$ in the daily life of patients with type 1 diabetes. This is very relevant because the better knowledge of the blood glucose behavior in patients under different types of intensive treatment can provide subsidies for therapeutic decisions and, thus, provide a better control of the disease [4-6].

Since the results of the DCCT (Diabetes Control and Complications Trial), a multicenter clinical study of 1441 volunteers with T1D, the indication of an intensive form of treatment has been considered fundamental to reduce the risks of the chronic microvascular complications of this disease [7]. In recent years, with the development of new analogues of insulin and continuous subcutaneous insulin infusion (CSII) systems, different treatment schemes have been used to conduct intensive insulin therapy $[6,8]$. However, this type of treatment increases the risk of severe hypoglycemia [9], an acute complication that may lead the patient to stop pursuing a more rigorous glycemic control or to present greater GV due to hyperglycemic rebound after hypoglycemia attacks. This is the reason why the present study aimed to evaluate the GV pattern in individuals with T1D under different types of intensive treatment and the correlations of this GV with different clinical and biochemical variables.

\section{Research design and methods}

\section{Participants and ethics}

The study was approved (294.635/2013) by the Research Ethics Committee of the Federal University of Uberlândia. All procedures

${ }^{*}$ Correspondence to: Ricardo Rodrigues, Faculty of Medicine, Federal University of Uberlândia, MG, Brazil, E-mail: ricardo.rodrigues@ufu.br

Key words: type 1 diabetes, glycemic variability, intensive treatment, insulin pump Received: January 03, 2020; Accepted: January 15, 2020; Published: January 20,2020 
were performed after participants had signed an informed consent term.

A cross-sectional study was done with volunteers with T1D ( $\mathrm{n}=90)$ assisted by the Diabetes Care Center of the Municipality of Uberlândia and the Clinical Hospital of the Federal University of Uberlândia.

For 2 years, 383 patients were attended in these facilities. Of these, 90 were part of the study because they fit into the inclusion criteria and did not present any exclusion factors. The inclusion criteria were: to have a diagnosis of type 1 diabetes and to be under intensive care with multiple daily doses of insulin (basal bolus scheme) or with continuous subcutaneous insulin infusion (CSII) system for at least 6 months. Exclusion criteria were: use of multivitamins, uncertain diagnosis of type of diabetes, severe chronic complications, febrile illness in the last month, skin changes that made sensor use impossible, blood dyscrasias, use of corticosteroids, heparin or oral anticoagulant. Patients who did not obtain the minimum number (5 to 7 ) of capillary glycemia to determine glycemic variability were also excluded from the study.

The volunteers were divided into 3 different groups according to the intensive treatment regimen: $\mathrm{G} 1(\mathrm{n}=54)$, basal $\mathrm{NPH}$ insulin and multiple daily doses of fast-acting insulin (bolus); G2 ( $n=19)$, basal insulin analog (glargine) and multiple daily doses of fast-acting insulin (bolus); and G3 ( $\mathrm{n}=17)$, CSII systems.

\section{Evaluation of glycemic control and glycemic variability}

Glycemic control was assessed by the glycated hemoglobin (HbA1c) levels over the past year, the estimated average glucose (eAG) and the daily average glucose (DAG). DAG was calculated from the records of self-monitoring blood glucose (SMBG), 5-7 times a day, using a blood glucometer (Accu-Chek Active ${ }^{\mathrm{Tw}}$, Roche Diagnostics, Indianapolis, IN, USA) and a management software (Accu-Chek $360^{\circ}$ diabetes management system, Roche Diagnostics, Indianapolis, IN, USA), or from the records of the continuous glucose monitoring (CGM) system, for 3 to 6 days, using the Guardian RT system ${ }^{\text {Tw }}$ (Medtronic, Northridge, CA, USA). GV was estimated from the standard deviation of the daily average glucose (SDDAG), the coefficient of variability (\%) of the daily average glucose $(\mathrm{CVDAG})$, given by $\mathrm{CVDAG}=(\mathrm{SDDAG} / \mathrm{DAG}) \times 100$, and the change in the levels of glycated hemoglobin $(\triangle \mathrm{HbA} 1 \mathrm{c})$, given by the difference between the highest and the lowest value of $\mathrm{HbAlc}$ over a year. HbAlc levels were assessed by high performance liquid chromatography (HPLC, Shimadzu, Kyoto, Japan).

\section{Other evaluations}

The data collected here also included anthropometric measurements, blood count, fasting plasma glucose (FPG), triglycerides (TGC), total-cholesterol (t-C), LDL-cholesterol (LDL-C) HDL-cholesterol (HDL-C), creatinine (Cr), microalbuminuria (MA) and thyroid stimulating hormone (TSH), in addition to the daily dose of insulin (DDI) used by each volunteer of the study. The probabilities of the volunteers developing hypoglycemia and hyperglycemia were estimated by the low blood glucose index (LBGI) and by the high blood glucose index (HBGI), which were obtained using the following equations:

$$
\begin{aligned}
& L B G I=\frac{1}{n} \sum_{i=1}^{n} r l\left(x_{i}\right)^{2}(1) \\
& H B G I=\frac{1}{n} \sum_{i=1}^{n} r h\left(x_{i}\right)^{2}
\end{aligned}
$$

\section{Statistical analysis}

The categorical variables were compared using the $\mathrm{x}^{2}$-test. The Shapiro-Wilk test was used to investigate the existence of normality in the data distribution. The comparisons of normal and non-normal variables among the different groups were done using ANOVA, followed by Bonferroni post-test, and the Kruskal-Wallis test, with DunnBonferroni post-test, respectively. Correlation analyzes were performed using the Pearson's or Spearman's test, when the distributions of the results were normal and non-normal, respectively. Differences associated with $\mathrm{p}$ values $\leq 0.05$ and $0.05<\mathrm{p}<0.06$ were considered statistically significant and borderline, respectively. All statistical analyzes were performed using the software Origin 2016 (Microcal, Northampton, MA, USA) and SPSS 15.0 (SPSS Inc., Chicago, IL, USA).

\section{Results}

The baseline characteristics of the study participants are summarized in Table 1. The total number of volunteers with T1D evaluated in this study is 90 (40 females, $44 \%$; and 50 males, 56\%).

Table 2 presents the comparison of the variables studied between types of intensive insulin therapy in volunteers with type 1 diabetes. There were no significant differences in age, sex ratio, FPG, t-C, HDL-C, TSH, MA, LBGI and HBGI. The group under continuous insulin infusion treatment presented lower levels of the glycemic control indicators $\mathrm{HbA1c}$, eAG and DAG and also lower values of the glycemic variability indicators SDDAG, CVDAG and $\triangle \mathrm{HbA} 1 \mathrm{c}$, in relation to the G1 and G2 groups. The group under treatment with basal NPH insulin and multiple daily doses of fast-acting insulin presented a borderline elevation in the DDI and blood levels of LDL-C in relation to other groups.

The relationships between the different variables were also investigated through correlation tests. The results obtained are shown in Table 3. Some significant correlations are worth mentioning. The glycemic control indicators $\mathrm{HbAlc}$, eAG and DAG had positive correlations with levels of TGC. DAG also presented a significant positive correlation with $\mathrm{HbAlc}$ (Figure 1) and, as would be expected, a positive correlation also with eAG. It is especially important to note that the indicator of glycemic variability SDDAG showed a significant positive correlation with the levels of HbA1c (Figure 2).

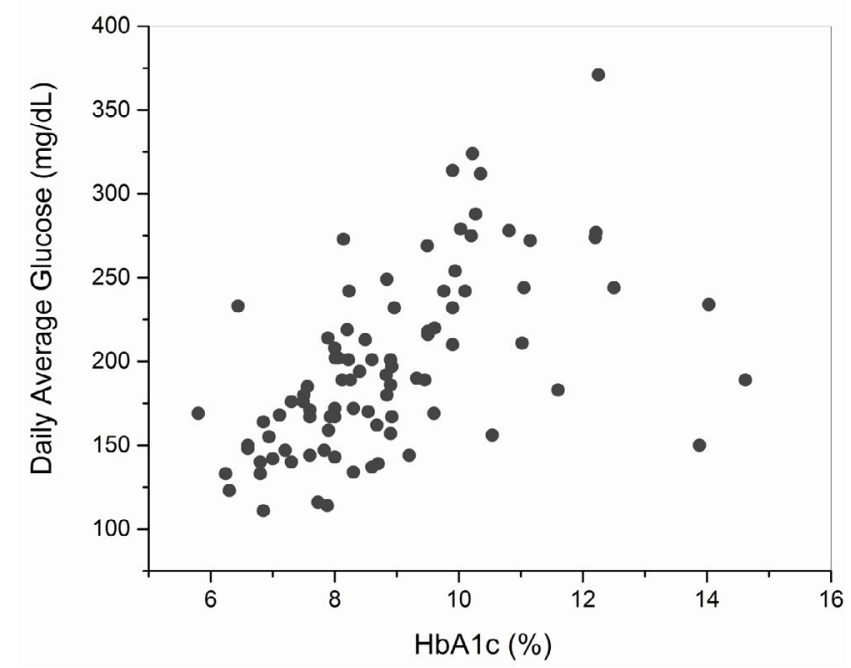

Figure 1. Dispersion diagram of the daily average glucose (DAG) and glycated hemoglobin $(\mathrm{HbA1c})$ in the studied population $(\mathrm{n}=90)$ 
Table 1. Baseline characteristics of the study population

\begin{tabular}{|c|c|c|c|c|}
\hline Variables & $\mathbf{N}$ & Median (IQR) & Min & Max \\
\hline Age (years) & 90 & $23.5(18-33)$ & 8 & 55 \\
\hline Body Mass Index $\left(\mathrm{kg} / \mathrm{m}^{2}\right)$ & 90 & $23(20.7-25)$ & 17 & 36.3 \\
\hline Duration of Diabetes (years) & 90 & $10(6-15)$ & 3 & 42 \\
\hline Daily Dose of Insulin (IU/kg) & 90 & $0.86(0.7-1.1)$ & 0.24 & 2.1 \\
\hline $\mathrm{HbA}_{1 \mathrm{c}}(\%)$ & 90 & $8.6(7.7-9.9)$ & 5.8 & 14.6 \\
\hline $\mathrm{HbA}_{1 \mathrm{c}}(\mathrm{mmol} / \mathrm{mol})$ & 90 & $69.5(61-85)$ & 40 & 136 \\
\hline$\Delta \mathrm{Hb}_{\mathrm{A} 1} \mathrm{c}$ & 90 & $0.99(0.6-1.4)$ & 0.13 & 4.54 \\
\hline Estimated Average Glucose (mg/dL) & 90 & $199(175-237)$ & 120 & 372 \\
\hline Daily Average Glucose (mg/dL) & 90 & $189(157-232)$ & 111 & 371 \\
\hline Standard Deviation of Daily Average Glucose (mg/dL) & 90 & $89.8(72-111)$ & 50 & 166.4 \\
\hline Coefficient of Variation of Daily Average Glucose (\%) & 90 & $47.3(40.1-53.9)$ & 23 & 90 \\
\hline Low Blood Glucose Index (\%) & 75 & $1.9(0.8-4.3)$ & 0.1 & 12.7 \\
\hline High Blood Glucose Index (\%) & 75 & $16.7(9.5-26.2)$ & 1.7 & 48.6 \\
\hline Fasting Plasma Glucose (mg/dL) & 90 & $153(101-220)$ & 44 & 390 \\
\hline Total Cholesterol (mg/dL) & 88 & $160.4(136.5-188.7)$ & 95 & 342 \\
\hline HDL-Cholesterol (mg/dL) & 88 & $52.1(16.6)$ & 16 & 94 \\
\hline LDL-Cholesterol (mg/dL) & 88 & $88(71-111)$ & 33 & 218.7 \\
\hline Triglycerides (mg/dL) & 88 & $83.9(55.4-122-2)$ & 35 & 379 \\
\hline Thyroid Stimulating Hormone (mIU/L) & 90 & $2.4(1.6-3.9)$ & 0.3 & 13.3 \\
\hline Microabuminuria ( $\mu \mathrm{g} / \mathrm{mg}$ Creatinine) & 89 & $4.9(2.0-11.3)$ & 0.8 & 758 \\
\hline
\end{tabular}

All variables were non-normally distributed and are presented as median (IQR), except HDL-C, which was normally distributed and is presented as mean (SD)

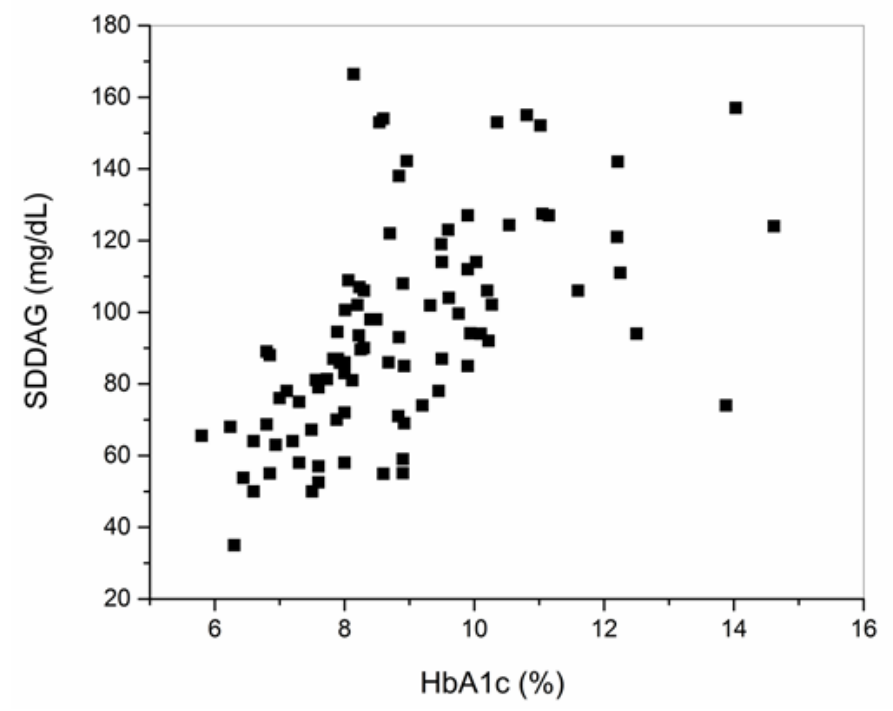

Figure 2. Dispersion diagram of the standard deviation of the daily average glucose (SDDAG) and glycated hemoglobin $(\mathrm{HbAlc})$ in the studied population $(\mathrm{n}=90)$

\section{Discussion and conclusions}

Glycemic variability in patients with type 1 diabetes, even under intensive treatment, is of great amplitude, and has direct correlations with the classic parameters of disease control, such as HbAlc and eAG, which are good risk indicators for the chronic complications of the disease.

The amplitude of glycemic variability in this study volunteers exceeded the acceptable tolerance range [2,10-12]. SDDAG values above $1 / 3$ of the mean or greater than $50 \mathrm{mg} /$ day and $\mathrm{CV}$ values greater than $36 \%$ are considered inadequate, since they represent a higher risk of severe hypoglycemia and elevation in the production of reactive oxygen species, factor associated with the generation of the cell injury that lead to the chronic complications of the disease [1].
The use of glycemic mean (DAG), standard deviation of the mean (SDDAG) and coefficient of variation of the mean (CV), determined by capillary glycemia or continuous interstitial glucose sensor as parameters for the quantification of glycemic variability was well established in recent studies [2,9,12-14], providing new tools to aid in therapeutic decisions. In the present study, the observation of a strong correlation between intraday glycemic variability and HbAlc, may allow the identification of patients who are more likely to fail in reaching the HbAlc goals in the coming months and thus allowing modification of treatment or early identification of their insulin needs.

The correlations observed in this study between glycemic variability parameters and $\mathrm{HbA} 1 \mathrm{c}$ and / or $\triangle \mathrm{HbAlc}$ are in agreement with the results reported in the ADAG study [2] for a population with T1D in which the glycemic mean was $172 \pm 37 \mathrm{mg} / \mathrm{dL}$ and SDDAG was $75 \pm 18.5$ $\mathrm{mg} / \mathrm{dL}$. That study reported the existence of correlations between SDDAG and other GV parameters such as the mean-amplitude glucose excursions (MAGE) and the continuous overall net glycemic action (CONGA) $(2 ; 10)$. When compared to individuals without diabetes, in which GV fluctuates between 0 and $55 \mathrm{mg} / \mathrm{dL}$ [15], patients with T1D showed GV about 2 to 3 times higher, which demonstrates the difficulty in achieving an insulin replacement system more similar to the physiological secretion pattern, which shows the need to develop new techniques and new parameters to minimize glycemic variability.

The use of $\triangle \mathrm{HbAlc}$, defined as the simple difference between the highest and the lowest $\mathrm{HbAlc}$, has recently been proposed as a parameter for the evaluation of the long-term effects of glycemic variability, in order to identify patients with chronic profile of greater glycemic instability, in order to predict the risk of development of the chronic complications of diabetes $[7,16,17]$. It is important to note that, in the present study, $\Delta \mathrm{HbA} 1 \mathrm{c}$ was significantly lower in the group under treatment with CSII.

The positive correlations of HbAlc, eAG and DAG with plasma levels of TGC are certainly resulting from lower activity of lipoprotein lipase in the liver, due to lower availability of insulin $[18,19]$. 
Table 2. Comparison of the studied variables* between types of intensive insulin therapy in volunteers with type 1 diabetes

\begin{tabular}{|c|c|c|c|c|c|c|}
\hline Variables & $\begin{array}{c}\text { G1 } \\
(\mathrm{n}=54)\end{array}$ & $\begin{array}{c}\text { G2 } \\
(\mathrm{n}=19)\end{array}$ & $\begin{array}{c}\text { G3 } \\
(\mathrm{n}=17)\end{array}$ & $\begin{array}{c}p \text {-Value } \\
\text { (G1\&G2) }\end{array}$ & $\begin{array}{c}p \text {-Value } \\
\text { (G1\&G3) }\end{array}$ & $\begin{array}{c}p \text {-Value } \\
\text { (G2\&G3) }\end{array}$ \\
\hline Age (years) & $23.5(19-33)$ & $24(20-36)$ & $23(13-35)$ & 0.693 & 0.686 & 0.375 \\
\hline Men/women (n/n) & $34 / 20$ & $10 / 9$ & $10 / 7$ & & & \\
\hline BMI $\left(\mathrm{kg} / \mathrm{m}^{2}\right)$ & $23.8(21-26)$ & $22.3(21-25)$ & $20(19-22)$ & 0.420 & 0.005 & 0.094 \\
\hline Duration of Diabetes (years) & $8(6-13)$ & $14(8-18)$ & $12(6-18)$ & 0.029 & 0.253 & 0.430 \\
\hline Insulin dose (IU/kg) & $0.92(0.7-1.1)$ & $0.88(0.7-1.1)$ & $0.71(0.6-0.8)$ & 0.909 & 0.017 & 0.057 \\
\hline $\mathrm{HbA}_{1 \mathrm{c}}(\%)$ & $8.9(8.2-10.1)$ & $8.2(7.8-9.3)$ & $7.5(6.8-7.9)$ & 0.191 & $<0.001$ & 0.009 \\
\hline $\mathrm{HbA}_{1 \mathrm{c}}(\mathrm{mmol} / \mathrm{mol})$ & $74(66-86)$ & $66(62-78)$ & $58(51-62)$ & 0.177 & $<0.001$ & 0.011 \\
\hline$\Delta \mathrm{HbA}_{1 \mathrm{c}}$ & $1.14 \pm(0.6-1.8)$ & $1.22(0.76-1.43)$ & $0.6(0.5-0.8)$ & 0.720 & 0.005 & 0.003 \\
\hline Estimated Average Glucose (mg/dL) & $209(188-243)$ & $190(180-221)$ & $169(148-181)$ & 0.211 & $<0.001$ & 0.008 \\
\hline Daily Average Glucose (mg/dL) & $205(169-242)$ & $172(155-232)$ & $167(147-176)$ & 0.162 & 0.001 & 0.097 \\
\hline Standard Deviation of Daily Average Glucose (mg/dL) & $100.1(83-119)$ & $88(76-127)$ & $64(57-72)$ & 0.487 & $<0.001$ & 0.001 \\
\hline Coefficient of Variation of Daily Average Glucose (\%) & $46.9(40.1-57.9)$ & $51.6(46.6-53.6)$ & $37.5(32.3-47.3)$ & 0.244 & 0.008 & 0.002 \\
\hline Low Blood Glucose (\%) & $1.7(0.8-4.2)$ & $1.8(0.9-3.8)$ & $4.3(0.6-6.6)$ & 0.937 & 0.201 & 0.272 \\
\hline High Blood Glucose (\%) & $15.8(9.5-25.4)$ & $19.2(16.5-22.3)$ & $10.4(5.8-27.7)$ & 0.440 & 0.641 & 0.368 \\
\hline Fasting Plasma Glucose (mg/dL) & $176.5(101-224)$ & $141(95-244)$ & $134(121-186)$ & 0.609 & 0.309 & 0.662 \\
\hline Total Cholesterol (mg/dL) & $173.4(136-197)$ & $150.3(136-175.5)$ & $160(137-176.7)$ & 0.118 & 0.288 & 0.757 \\
\hline HDL-Cholesterol (mg/dL) & $49.4(15.1)$ & $54.5(16.8)$ & $58.6(20)$ & & & \\
\hline LDL-Cholesterol (mg/dL) & $97.3(77.2-118.6)$ & $80.7(64.6-98.9)$ & $79(66-108)$ & 0.038 & 0.108 & 0.810 \\
\hline Triglycerides (mg/dL) & $92(67-140)$ & $87(55-112)$ & $55(48-76)$ & 0.276 & 0.001 & 0.050 \\
\hline Thyroid Stimulating Hormone (mIU/L) & $2.8(1.7-4.0)$ & $2.4(1.8-5.4)$ & $1.8(1.1-2.8)$ & 0.927 & 0.040 & 0.073 \\
\hline Microalbuminuria (mcg/mg Creatinine) & $4.7(1.9-8.8)$ & $4(2.4-8.4)$ & $11.4(2.7-58.4)$ & 0.971 & 0.061 & 0.120 \\
\hline
\end{tabular}

Except for HDL-C, which was normally distributed and is presented as mean (SD), all other variables were non-normally distributed and are presented as median (IQR). Comparisons were done by ANOVA with Bonferroni post-hoc test for HDL-C and by Kruskall-Wallis with Dunn-Bonferroni post-hoc test for all other variables

Table 3. Spearman's $\sigma$ coefficients for the correlations between all pairs of variables studied

\begin{tabular}{|c|c|c|c|c|c|c|c|c|c|c|c|c|c|c|c|c|c|c|c|c|}
\hline$\#$ & Variables & 1 & 2 & 3 & 4 & 5 & 6 & 7 & 8 & 0 & 10 & 11 & 12 & 13 & 14 & 15 & 16 & 17 & 18 & 19 \\
\hline 1 & SDDAG & 1.00 & & & & & & & & & & & & & & & & & & \\
\hline 2 & DAG & $0.64^{\S}$ & 1.00 & & & & & & & & & & & & & & & & & \\
\hline 3 & $\Delta \mathrm{HbA} 1 \mathrm{c}$ & $0.42^{\S}$ & $0.32^{\S}$ & 1.00 & & & & & & & & & & & & & & & & \\
\hline 4 & $\mathrm{HbA} 1 \mathrm{c}$ & $0.67^{\S}$ & $0.64^{\S}$ & $0.41^{\S}$ & 1.00 & & & & & & & & & & & & & & & \\
\hline 5 & eAG & $0.67^{\S}$ & $0.65^{\S}$ & $0.41^{\S}$ & $1.00^{\S}$ & 1.00 & & & & & & & & & & & & & & \\
\hline 6 & $\mathrm{CV}$ & $0.55^{\S}$ & $-0.23^{*}$ & $0.27^{*}$ & 0.10 & 0.11 & 1.00 & & & & & & & & & & & & & \\
\hline 7 & FPG & 0.13 & $0.21^{*}$ & $0.28^{8}$ & $0.36^{\S}$ & $0.37^{\S}$ & -0.07 & 1.00 & & & & & & & & & & & & \\
\hline 8 & LBGI & 0.03 & 0.09 & 0.05 & 0.06 & 0.06 & -0.02 & -0.08 & 1.00 & & & & & & & & & & & \\
\hline 19 & HBGI & 0.07 & -0.15 & -0.03 & -0.08 & -0.08 & 0.22 & 0.05 & $-0.28^{*}$ & 1.00 & & & & & & & & & & \\
\hline 10 & Age & -0.11 & -0.15 & -0.13 & $-0.21^{*}$ & -0.20 & 0.05 & -0.15 & 0.07 & 0.04 & 1.00 & & & & & & & & & \\
\hline 11 & BMI & 0.02 & -0.15 & 0.08 & 0.06 & 0.06 & 0.19 & 0.12 & $-0.27^{*}$ & $0.28^{*}$ & $0.43^{\S}$ & 1.00 & & & & & & & & \\
\hline 12 & Disease Duration & 0.03 & -0.16 & -0.05 & -0.14 & -0.13 & 0.20 & -0.08 & 0.07 & -0.01 & $0.60^{\S}$ & $0.24^{*}$ & 1.00 & & & & & & & \\
\hline 13 & Dose & $0.25^{*}$ & $0.39^{\S}$ & $0.24^{*}$ & $0.25^{*}$ & $0.25^{*}$ & -0.11 & 0.12 & -0.07 & -0.17 & $-0.50^{\S}$ & -0.16 & $-0.29^{\S}$ & 1.00 & & & & & & \\
\hline 14 & Microalbuminuria & -0.13 & -0.03 & 0.14 & 0.09 & .086 & -0.12 & $0.22^{*}$ & $0.25^{*}$ & -0.12 & 0.02 & -0.05 & 0.18 & 0.02 & 1.00 & & & & & \\
\hline 15 & $\mathrm{t}-\mathrm{C}$ & -0.04 & 0.14 & 0.00 & $0.22^{*}$ & $0.22^{*}$ & $-0.22^{*}$ & $0.31^{\S}$ & 0.01 & -0.05 & 0.14 & $0.23^{*}$ & -0.03 & 0.08 & $0.26^{*}$ & 1.00 & & & & \\
\hline 16 & TGC & 0.19 & $0.27^{*}$ & $0.31^{\S}$ & $0.40^{\S}$ & $0.40^{\S}$ & -0.09 & $0.40^{\S}$ & -0.11 & 0.05 & 0.01 & $0.27^{*}$ & -0.08 & $0.29^{\S}$ & 0.15 & $0.48^{\S}$ & 1.00 & & & \\
\hline 17 & LDL-C & 0.01 & 0.14 & 0.03 & 0.20 & 0.20 & -0.11 & $0.28^{\S}$ & -0.05 & -0.06 & 0.01 & $0.28^{\S}$ & -0.07 & 0.16 & 0.21 & $0.85^{\S}$ & $0.40^{\S}$ & 1.00 & & \\
\hline 18 & HDL-C & -0.14 & -0.12 & -0.19 & -0.12 & -0.12 & -0.09 & -0.06 & 0.08 & 0.03 & 0.12 & -0.17 & 0.06 & -0.21 & -0.07 & $0.25^{*}$ & $-0.33^{\S}$ & -0.08 & 1.00 & \\
\hline 19 & TSH & 0.09 & 0.19 & -0.05 & 0.07 & 0.08 & -0.06 & 0.14 & -0.03 & 0.12 & 0.01 & 0.12 & -0.07 & 0.09 & -0.05 & 0.06 & $0.30^{\S}$ & 0.14 & $-0.31^{\S}$ & 1.00 \\
\hline
\end{tabular}

${ }^{*} \mathrm{p}<0.05$ and ${ }^{\S} \mathrm{p}<0.01$

In relation to treatments, only the intensive treatment group with CSII approached these goals of good disease control, with a low GV and $\mathrm{HbAlc}$ close to 7\%. Among the possible reasons for this improved performance of the group under treatment with CSII is the use of fast acting insulin analogue for both basal and meal coverage (bolus dose), while other forms of intensive treatment always combine one slowacting insulin with fast-acting insulin applications. It is well known that intermediate and slow-acting insulin, mainly NPH insulin, presents great intra-individual pharmacokinetic variability, which can justify greater fluctuation in blood glucose [20,21]. Another important reason for that difference is the way in which insulin is administered; fractional and gradual release in CSII better mimics the physiological pancreatic secretion in relation to other systems, without the deposition of higher amount of slow acting insulin in the subcutaneous tissue as occurs in those systems. In fact, the use of significantly lower doses of insulin in the volunteers being treated with CSII is consistent with the best results presented by the disease control indicators.

The limitations of this study are those of real-life studies, where the groups are not controlled and therefore what determines the therapeutic choice used by each patient group could constitute a selection bias. However, the real-life studies are important because they contribute with clinical answers on the effectiveness of a treatment or intervention in routine situations and, with this, allow high generalization of the findings [22]. 
It is important to note that the population of the present study was fully constituted of volunteers attended by government public assistance services, with strict criteria to define the type of treatment to be used. According to these criteria, the use of basal insulin analog (glargine) associated to multiple daily doses of fast-acting insulin (G2) is only authorized by the Brazilian Public Health System after therapeutic failure with the scheme based in basal NPH insulin and multiple daily doses of fast-acting insulin (G1). Besides this, the use of the CSII system (G3) is only released after therapeutic failure with the others two types of intensive treatment, due to the high cost of this therapy. Therefore, very possibly group 3 was composed of patients with more difficult clinical conditions in relation to groups 2 and 1 . The higher values of microabuminuria, with a borderline significance, of group 3 in relation to group 1, seem to support this idea.

Due to the multifactorial nature of the mechanisms involved in the pathogenesis of the chronic complications of diabetes, which include ischemia, inflammation and glycotoxicity, it was expected to find weak to moderate correlations between the variables studied. However, the existence of these correlations reinforces the need to develop new and more specific studies on the implications of glycemic variability in the pathogenesis of the chronic T1D complications.

Similar to what was found in the literature with controlled studies, in this real-life study greater efficacy was observed in the glycemic control of the group with continuous subcutaneous insulin infusion, which had values closer to the goal of good control of HbAlc and lower glycemic variability in relation to the other types of intensive treatment analyzed here.

In addition, the positive correlation observed between SDDAG and $\mathrm{HbA1c}$ indicates that this GV marker can be used in the short term as a predictor of the glycated hemoglobin levels in order to allow directions and earlier adaptations in clinical management.

\section{Duality of interest}

The authors confirm that there is no duality of interest associated with this manuscript.

\section{Author contributions}

R.R. performed the study design, collected data and wrote the draft manuscript. L.A.M. collected data and helped in the edition of the manuscript. M.S.G-F. and M.B.N. performed the statistical analysis and helped in the preparation of the manuscript draft. P.T.J. and E.S.R. helped in the study design and in the discussion of the manuscript. N.P-S. collaborated in the design of the study, in the discussion of its results and in the final edition of the manuscript.

\section{Funding}

This study received financial support (APQ-03602-13) of 'Fundação de Apoio à Pesquisa de Minas Gerais (FAPEMIG) for the acquisition of the CGM system and the sensors used during its implementation. N. Penha-Silva was supported by a scientific productivity grant from "Conselho Nacional de Pesquisa e Desenvolvimento" (CNPq).

\section{References}

1. Brownlee M (2005) The pathobiology of diabetic complications: a unifying mechanism Diabetes 54: 1615-1625. [Crossref]

2. Borg R, Kuenen JC, Carstensen B, Zheng H, Nathan DM, et al. (2010) Associations between features of glucose exposure and A1C: the A1C-Derived Average Glucose (ADAG) study. Diabetes 59: 1585-1590. [Crossref]

3. Kim SK, Suh S, Kim MY, Chung HS, Hur KY, et al. (2011) Three-day continuous glucose monitoring for rapid assessment of hypoglycemic events and glycemic variability in type 1 diabetic patients. Endocr J 58: 535-541.

4. Breton M, Farret A, Bruttomesso D, Anderson S, Magni L, et al. (2012) Fully integrated artificial pancreas in type 1 diabetes: modular closed-loop glucose control maintains near normoglycemia. Diabetes 61: 2230-2237.

5. Breton MD, Kovatchev BP (2010) Impact of blood glucose self-monitoring errors on glucose variability, risk for hypoglycemia, and average glucose control in type 1 diabetes: an in silico study. J Diabetes Sci Technol 4: 562-570.

6. Garvey K, Wolfsdorf JI (2015) The impact of technology on current diabetes management. Pediatr Clin North Am 62: 873-888.

7. Kilpatrick ES, Rigby AS, Atkin SL (2008) A1C variability and the risk of microvascular complications in type 1 diabetes: data from the Diabetes Control and Complications Trial. Diabetes Care 31: 2198-2202.

8. Prieto-Tenreiro A, Villar-Taibo R, Pazos-Couselo M, González-Rodríguez M Casanueva F, et al. (2012) [Benefits of subcutaneous continuous insulin infusion in type 1 diabetic patients with high glycemic variability]. Endocrinol Nutr 59: 246-253.

9. Gubitosi-Klug RA, Braffett BH, White NH, Sherwin RS, Service FJ, et al. (2017) Risk of Severe Hypoglycemia in Type 1 Diabetes Over 30 Years of Follow-up in the DCCT/ EDIC Study. Diabetes Care 40: 1010-1016. [Crossref]

10. Service FJ (2013) Glucose variability. Diabetes 62: 1398-1404. [Crossref]

11. Monnier L, Colette C, Wojtusciszyn A, Dejager S, Renard E, et al. (2017) Toward defining the threshold between low and high glucose variability in diabetes. Diabetes Care 40: 832-838. [Crossref]

12. Rodbard D (2009) Interpretation of continuous glucose monitoring data: glycemic variability and quality of glycemic control. Diabetes Technol Ther 1: 55-67.

13. Baghurst PA, Rodbard D, Cameron FJ (2010) The minimum frequency of glucose measurements from which glycemic variation can be consistently assessed. J Diabetes Sci Technol 4: 1382-1385.

14. DeVries JH (2013) Glucose variability: where it is important and how to measure it. Diabetes 62: 1405-1408. [Crossref]

15. Hill NR, Oliver NS, Choudhary P, Levy JC, Hindmarsh P, et al. (2011) Normal reference range for mean tissue glucose and glycemic variability derived from continuous glucose monitoring for subjects without diabetes in different ethnic groups. Diabetes Technol Ther 13: 921-928.

16. Marcovecchio ML, Dalton RN, Chiarelli F, Dunger DB (2011) A1C variability as an independent risk factor for microalbuminuria in young people with type 1 diabetes. Diabetes Care 34: 1011-1013. [Crossref]

17. Kilpatrick ES, Rigby AS, Atkin SL (2009) Effect of glucose variability on the long-term risk of microvascular complications in type 1 diabetes. Diabetes Care 32: 1901-1903.

18. Fick T, Jack J, Pyle-Eilola AL, Henry RK (2017) Severe hypertriglyceridemia at new onset type 1 diabetes mellitus. J Pediatr Endocrinol Metab 30: 893-897. [Crossref]

19. Dunn FL (1992) Plasma lipid and lipoprotein disorders in IDDM. Diabetes 41: 102 106. [Crossref]

20. Bolli GB (1989) The pharmacokinetic basis of insulin therapy in diabetes mellitus Diabetes Res Clin Pract 6: 3-15.

21. Heinemann L (2002) Variability of insulin absorption and insulin action. Diabetes Technol Ther 4: 673-682.

22. Saturni S, Bellini F, Braido F, Paggiaro P, Sanduzzi A, et al. (2014) Randomized controlled trials and real life studies. approaches and methodologies: a clinical point of view. Pulm Pharmacol Ther 27: 129-138.

Copyright: (C2020 Rodrigues R. This is an open-access article distributed under the terms of the Creative Commons Attribution License, which permits unrestricted use, distribution, and reproduction in any medium, provided the original author and source are credited. 